\begin{tabular}{|c|c|c|c|c|c|c|}
\hline \multicolumn{7}{|c|}{ Abstract OC-019 Table 1} \\
\hline Station & $\begin{array}{l}\text { Cardia } \\
(\%)\end{array}$ & $\begin{array}{l}\text { Fundus } \\
(\%)\end{array}$ & $\begin{array}{l}\text { Body } \\
(\%)\end{array}$ & $\begin{array}{l}\text { Incisura } \\
(\%)\end{array}$ & $\begin{array}{l}\text { Antrum } \\
(\%)\end{array}$ & $\begin{array}{l}\text { Pylorus } \\
(\%) \\
\end{array}$ \\
\hline $\begin{array}{l}\text { Fundal dependent + } \\
\text { antral dependent }\end{array}$ & 87 & 99 & 99 & 100 & 100 & 45 \\
\hline $\begin{array}{l}\text { Fundal dependent }+ \\
\text { opposite antral dependent }\end{array}$ & 92 & 99 & 99 & 100 & 100 & 86 \\
\hline
\end{tabular}

Pyloric canal vector surface point

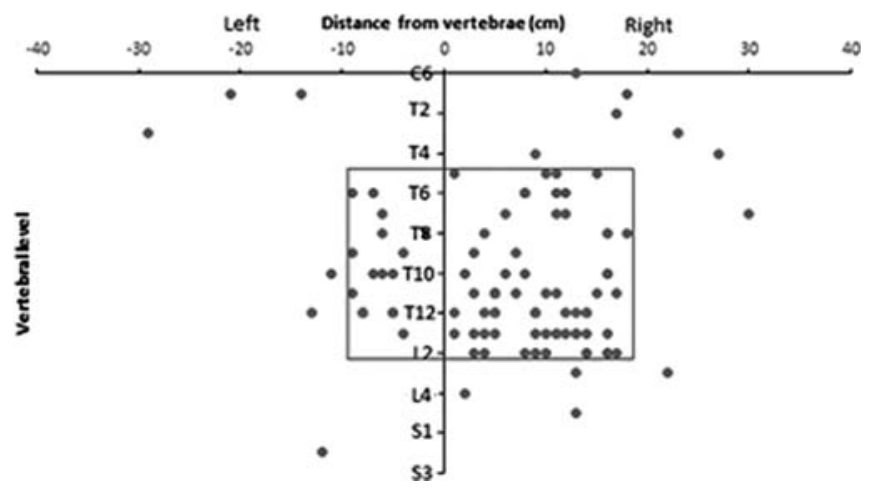

Abstract OC-019 Figure 1

ability of a capsule endoscope to visualise 6 anatomical landmarks (cardia, fundus, body, incisura, antrum and pylorus). Success of visualisation of an anatomical area was only accepted when $>90 \%$ mucosal visualisation was achieved from a particular station. The pyloric canal angles were calculated to create a vector. We mapped the position of this vector on the patient's skin (pyloric canal vector surface point) to determine the optimal placement of the magnet that would allow traversing of the capsule endoscope through the pylorus.

Results There were 65 female and 35 male patients. Mean age of patients was 53 years (s.d+/-18 years). Best mucosal visualisation of the stomach landmarks was achieved from 3 stations; fundal dependant, antral dependent and opposite the antral dependent points. Maximal visualisation of the whole of the stomach, required combining 2 stations as shown in Table 1.

The box in the figure shows the placement of the magnet in the upper back towards the right loin would allow pyloric traversing of the capsule endoscope in $83 \%$ of cases. Increasing age $(\mathrm{p}=0.03)$ and inability to view the pylorus $(\mathrm{p}=0.04)$ were predictors of being outside the box.

Conclusion CT modelling has provided important data regarding the optimal stations in the stomach to position a magnetic capsule endoscope to allow maximal luminal mucosal visualisation and traversing the pylorus. Although there is some extreme variation in the upper GI anatomy, the majority of cases will allow the use of a single standard method in performing MACE which may be very useful for screening purposes.

Disclosure of Interest None Declared.

\section{OC-020 COMPARATIVE EFFECTIVENESS OF NOVEL TECHNIQUES FOR BARRETT'S OESOPHAGUS (BO) SCREENING IN THE COMMUNITY: A PROSPECTIVE RANDOMISED TRIAL}

${ }^{1} \mathrm{SS}$ Sami ${ }^{*},{ }^{2} \mathrm{KT}$ Dunagan, ${ }^{2} \mathrm{ML}$ Johnson, ${ }^{3} \mathrm{CD}$ Schlek, ${ }^{3} \mathrm{AR}$ Zinsmeister, ${ }^{2} \mathrm{LM}$ Wong Kee Song, ${ }^{2} \mathrm{KK}$ Wang, ${ }^{2} \mathrm{DA}$ Katzka, ${ }^{1} \mathrm{~K}$ Ragunath, ${ }^{2} \mathrm{PG}$ lyer. ${ }^{1}$ Digestive Diseases Centre and NIHR Biomedical Research Unit, University of Nottingham, UK, Nottingham, UK; ${ }^{2}$ Gastroenterology and Hepatology, Mayo Clinic, Rochester, MN, USA; ${ }^{3}$ Biomedical Statistics and Informatics, Mayo Clinic, Rochester, MN, USA

10.1136/gutjnl-2014-307263.20
Introduction $\mathrm{BO}$ is the strongest precursor of oesophageal adenocarcinoma. Participation patterns and effectiveness of $\mathrm{BO}$ community screening using unsedated transnasal endoscopy (uTNE) is unknown. Feasibility of mobile van screening closer to home is also unknown. We aimed to assess the effectiveness of this technique compared to sedated endoscopy (SE).

Methods A population cohort $\geq 50$ years of age, with no history of endoscopic evaluation, was identified from a group of subjects who previously completed a validated symptom questionnaire. Patients were randomised (stratified by age, sex and reflux symptoms) and invited to undergo either uTNE in a mobile research van (muTNE), uTNE in outpatient endoscopy suite (huTNE) or SE. uTNE was performed using a portable oesophagoscope with a disposable sheath. Procedure performance characteristics and validated tolerability scales $(0=$ none and $10=$ severe $)$ were recorded.

Results 459 subjects were contacted and 209 (46\%) agreed to undergo study procedures (muTNE $\mathrm{n}=76$, huTNE $\mathrm{n}=72$, SE $\mathrm{n}=61$ ). Baseline characteristics were comparable among the three groups.

Participation rates were numerically higher in the unsedated arms (muTNE 47.5\%, huTNE 45.7\%) than in the SE arm $(40.7 \%)(p=0.27)$. Patients with acid reflux symptoms $\geq 1$ / week were more likely to participate (odds ratio 2.94 , 95\% confidence interval 1.47, 5.88).

Complete evaluation of the oesophagus was comparable using muTNE (99\%), huTNE (96\%) and SE (100\%) techniques. Successful biopsy acquisition was lower in the muTNE (79\%) and huTNE $(83 \%)$ groups compared to SE $(100 \%)(p=0.001)$.

Mean duration (minutes) of examination was shorter in the SE arm (4.7) than in muTNE (8.0) and huTNE (8.5) groups (p $<0.001)$. However, recovery time was much longer for SE (67.3) compared to muTNE (15.5) and huTNE (18.5) techniques $(\mathrm{p}<0.001)$.

While overall tolerability for SE was better than muTNE and huTNE (mean score 0.4 vs. 1.9 and 2.2 respectively, $\mathrm{p}<0.001$ ), the majority of patients who underwent muTNE and huTNE were willing to undergo the same procedure again in future (79\% and 83\%, respectively). No serious adverse events were reported. 16 subjects (7.6\%) were diagnosed with BO.

Conclusion In this first large randomised trial evaluating novel approaches for community screening for $\mathrm{BO}$, unsedated mobile van and clinic screening with TNE was feasible and effective. The patients' visit was significantly shorter with adequate tolerability, acceptability and safety profiles. Mobile and outpatient techniques may provide a cost-effective alternative to SE for BO screening.

Disclosure of Interest S. Sami: None Declared, K. Dunagan: None Declared, M. Johnson: None Declared, C. Schlek: None Declared, A. Zinsmeister: None Declared, L. M. Wong Kee Song: None Declared, K. Wang: None Declared, D. Katzka: None Declared, K. Ragunath Grant/research support from: Olympus (keymed, UK) and Intromed Ltd. (Seoul, South Korea), P. Iyer: None Declared.

\section{OC-021 GASTRIC ULCER FOLLOW-UP: THE IMPACT OF NICE GUIDELINES}

R Cochrane, S Thanaraj, A Sainsbury, C Selinger*. Gastroenterology, Leeds Teaching Hospitals NHS Trust, Leeds, UK

\subsection{6/gutjnl-2014-307263.21}

Introduction While the National Institute for Health and Care Excellence (NICE) recommends a follow-up gastrocopy 
(FU-OGD) within 8 weeks for all gastric ulcers to exclude malignancy, internationally this recommendation is controversial. This audit examined the impact of this NICE guideline.

Methods All patients with a first endoscopic diagnosis of gastric ulcer between January 2012 and September 2013 at this large teaching hospital were included. Patients with known gastric ulcers prior to the study period or those referred for tertiary assessment were excluded. Patient demographics, data on endoscopic appearance and histological reports were recorded. We analysed endoscopic assessments, compliance with NICE guidance and the malignancy yield.

Results 432 patients (53\% male) with a mean age of 65 years presented with a gastrointestinal bleed (38\%), anaemia (13\%), dyspepsia (18\%) or other symptoms (31\%). 142 patients were not biopsied initially (73\% GI bleed, 6\% anticoagulation, 21\% no reason). Of these 96 received FU-OGD for biopsies after a median of 7 days. 290 patients had an average of 4.8 biopsies taken on index endoscopy. Of these 194 underwent repeat OGD after a median of 70 days. Only 33\% underwent FU-OGD within the recommended time frame. Reasons for not undergoing FU-OGD were failure to request (10\%), deemed medically inappropriate (98\%), death before FU-OGD due (12\%), patient non-attendance (24\%), malignancy on 1st biopsy (9\%) and unknown (26\%). Ulcer healing was found in 236 patients. Gastric neoplasia was found in 27 patients (19 adenocarcinomas, 2 cases of dysplasia, 5 lymphomas, 1 melanoma) leading to a diagnosis of malignancy in $6 \%$ of patients with gastric ulcers. Of these 25 (93\%) were diagnosed on first biopsy. After excluding those patients without follow-up, the cancer yield of FU-OGD after initially benign biopsy was 2 of 225 patients (0.9\%).

Conclusion In this large cohort of patients with gastric ulcers $6 \%$ were found to have malignant disease. Of these over $90 \%$ were diagnosed with first biopsy, highlighting the need for all gastric ulcers to be biopsied. For many patients FU-OGD was deemed inappropriate. A high non-attendance rate was also noted, which may relate to the uncomfortable and invasive nature of endoscopy. While rapid FU-OGD for biopsies was achieved in those with no initial biopsies, many patients waited longer than the recommended 8 weeks after initial benign biopsies. However, the cancer yield of FU-OGD after benign biopsies remains low. Strategies to reduce the burden of FU-OGD for both patients and the health service would be highly desirable.

Disclosure of Interest R. Cochrane: None Declared, S. Thanaraj: None Declared, A. Sainsbury: None Declared, C. Selinger Grant/ research support from: Ferring, Nycomed, Shire, Warner Chilcott.

\section{Small bowel and nutrition free papers}

\section{OC-022 DEVELOPMENT AND OUTCOME OF INTESTINAL FAILURE IN CROHN'S DISEASE: 3 DECADES OF EXPERIENCE FROM A NATIONAL REFERRAL CENTRE}

E Nixon*, P Allan, S Sidhu, A Abraham, A Teubner, G Carlson, S Lal. Intestinal Failure Unit, Salford Royal NHS Foundation Trust, Salford, UK

10.1136/gutinl-2014-307263.22

Introduction Intestinal Failure (IF) is a rare complication of Crohn's disease (CD) which carries significant morbidity, for which there is a relative paucity of data. We describe a large series of patients with CD and IF and evaluate factors associated with IF development.

Methods This was a retrospective study from a prospectively maintained database of patients referred to a national IF centre.
Patients with CD were identified if on home parenteral nutrition (PN) for at least 12 months in the period between 1980-2011, and case notes were reviewed. Severe IF was defined as onset of IF within 15 years of CD diagnosis. Comparison of frequencies was performed using the Chi-squared test. Multivariate logistic regression models were used to identify independent associated factors after univariate analysis.

Results 78 patients were identified. The median time from CD diagnosis to commencing PN was 120 (12-552) months. Median time from CD diagnosis to first surgery was $36(0-312)$ months. Patients underwent a median of 3 (1-7) small bowel (SB) resections prior to commencing $\mathrm{PN}$, and the median remnant $\mathrm{SB}$ length was $130(40-375) \mathrm{cm}$. Patients underwent a total of 312 operations but data were unavailable for 3 of these. The primary indication for the first surgery was stricturing $(61.5 \%)$, followed by penetrating $(23.1 \%)$ and inflammatory $(15.4 \%)$ disease. Operative complications were higher in patients undergoing emergency $(37 / 85 ; 43.5 \%)$ vs. elective $(53 / 224 ; 23.7 \%)$ procedures $(\mathrm{p}$ $=0.001)$ and in surgeries performed outside $(87 / 255 ; 34.1 \%)$ vs. within $(3 / 54 ; 5.6 \%)$ the IFU $(\mathrm{p}<0.0001))$.

234 subsequent operations were undertaken after the first, but indication data were missing in 7 operations; operative complications were the primary indication for surgical intervention in (76/227) $33.5 \%$ of cases after the first surgery. In multivariate analysis, severe IF was associated with surgical complications ( $p$ $=0.003)$, higher number of SB resections $(\mathrm{p}=0.005)$ and earlier age of $C D$ diagnosis $(\mathrm{p}=0.005)$.

25/78 (32.1\%) patients achieved nutritional autonomy during a median follow-up of 36 (12-376) months after commencing PN. There were 68 documented catheter infections $(0.42$ infections/1000 catheter days) in this patient cohort.

9 patients died during follow-up as a result of liver disease ( $\mathrm{n}$ $=3)$, complex $\mathrm{CD} /$ overwhelming sepsis $(\mathrm{n}=2)$ or other causes $(\mathrm{n}=4)$.

Conclusion Recurrent surgeries with operative complications are significant factors leading to severe IF. These are important considerations in guiding therapeutic decisions in $\mathrm{CD}$, given the morbidity associated with chronic IF.

Disclosure of Interest None Declared.

\section{OC-023 FACTORS INFLUENCING MORTALITY FOLLOWING GASTROSTOMY INSERTION}

RE Andrews*, M Kurien, JS Leeds, J Grant, ME McAlindon, DS Sanders. Gastroenterology, Royal Hallamshire Hospital, Sheffield, UK

\subsection{6/gutjnl-2014-307263.23}

Introduction High mortality rates have previously been reported following gastrostomy insertion, particularly among certain patient groups (e.g., dementia). With the increasing use of prophylactic gastrostomy for head and neck cancer (HNC), our group aimed to examine survival in this cohort compared to other referral indications and assess risk factors.

Methods Gastrostomy insertions were examined from two hospitals in Sheffield between 2004-2013. Data was prospectively collected from all referred patients including demographic data, biochemical parameters, referral indications and gastrostomy type. Statistical analysis was performed with Chi-squared or Fishers exact tests.

Results 1733 patients were included (1004 male, mean age $=65)$. 30 day and 1 year mortality was $9.66 \%$ and $44.98 \%$ respectively. Indications for gastrostomy included; HNC (n = 591), neurological disease $(\mathrm{n}=429)$, dysphagic stroke $(\mathrm{n}=$ 